\title{
Challenges in the Introduction of Nation-Wide School-Based Vaccination against HPV in Peru
}

\author{
Mary Edith Penny*, Gabriela Anaya, Kristel Avila, VariniaArevalo and Rosario M Bartolini
}

${ }^{1}$ Institute of Investigation Nutritional, Ave La Molina 1885, La Molina, Lima 12, Peru.

Received: May 31, 2018; Accepted: June 25, 2018; Published: August 06, 2018

*Corresponding authors: Mary Edith Penny, Institute of Investigation Nutritional, Ave La Molina 1885, La Molina, Lima 12, Peru, E-mail: mpenny@iin.sld.pe

\begin{abstract}
Cancer of the cervix can now be prevented by vaccination against human papilloma virus. Demonstration projects have been successful but there are few reports of the challenges related to national school based programs. In conjunction with the Ministry of Health we conducted interviews and a household survey in three contrasting areas of Peru to obtain the perspective of different key actors. Of grade eligible girls $66 \%$ had received at least one dose, but $14 \%$ had discontinued. The main challenges were logistic: ensuring that that all schools participate; calculating the number of eligible girls; fitting the 3 doses into a 9m school year; written parental consent; coordination with schools; arrangements for a mopping-up strategy; combating discontinuation; reaching the distant and sparsely populated areas; and the additional work burden for health personnel. A lack of media information and educational materials left parents ill-informed but vaccine refusal was unusual. A two dose strategy will reduce the work load and cost but will not solve all the logistics problems. Ensuring an early start to the campaign based on estimated needs; a communication strategy to increase girls and parental motivation and community involvement are likely to increase coverage. Taking advantage of existing parent school interactions and considering "optout"would facilitate the consent process and, if acceptable, should be considered.
\end{abstract}

Keywords: Human Papilloma Virus; HPV Vaccine; Health Services, Implementation; Schools; Acceptance; Program Evaluation; Peru;

\section{Introduction}

Cancer of the cervix, the most common cause of cancer related deaths in women aged 15-44 years can now be prevented with vaccines against the human papilloma virus [1].Two vaccines are licensed and now part of the national strategy in 64 countries [2] and reductions in cytological precursors of cancer have been reported $[3,4,5]$. In countries where the majority of children are in school, school-based vaccination programs have been the intervention of choice and these are now in place in many countries [6]. Some studies have examined these school based programs, comparing with health service based vaccination $[7,8]$ and have generally found them effective, often achieving more than $80 \%$ coverage. However, few have been in low or middle income countries, especially Latin America, and with one exception, a state wide HPV vaccination program through schools in Brazil, [9] these have been demonstration project or GAVI assisted programs.
In Peru a demonstration school-based vaccination successfully vaccinated $85 \%$ of girls [10] and in 2011 a national strategy of school based vaccination was started, problems with supply interrupted the program at first, but vaccination was renewed in 2015. We have undertaken a study to understand the factors that have determined vaccine uptake in the context of a recently starting national level school based program.

\section{Methods}

\section{Design of the Study}

The study was designed as an exploratory mixed-methods cross-sectional study to understand the factors that facilitated or hindered the performance of school-based HPV vaccination in Peru.

Following consultation with the Ministry of Health (MOH) we used a purposive sampling strategy to identify regions with varying reported coverage that included three geographically and socially different regions encompassing Amazon Rain Forest (Ucayali) ,mountains (Cusco) and coastal cities (Callao a province of Lima) and represented the challenge of implementing a schoolbased vaccination strategy.

Within each region, in discussion with the health authority, we purposefully selected two contrasting districts, in Ucayali, one rural and one urban, and in Cusco two different geographical areas. In each region we selected two health facilities that provided diverse vaccination scenarios and coverage, and within the catchment area of each health facility we selected one primary school. In Callao, which is entirely urban, we selected one district and two health facilities. In total we included 5 districts and 10 health facilities and schools.

\section{Data Collection}

\section{Interview subjects}

The study included in depth interviews to key actors at different levels of decision making including the National director, regional, district and local health facility vaccine strategy personnel. Two local public schools in each district were visited and the headmasters/mistresses, and the teachers of at least one 5 th grade classes were interviewed.

To obtain information about the vaccination process in the 
school we asked teachers of 2-3 classes to select girls who had been vaccinated, and following parental consent we conducted focus groups with 5-6 girls. We used storytelling, drawing and discussion with the girls to understand the organization, processes and the reaction of the girls to the vaccination.

In total we interviewed 47 health personnel, 31 teachers, 14 mothers (excluding the survey) and 59 girls in focus groups and 6 representatives of the media, or people considered influential in the society.

\section{Interview content}

Interview guides were developed following specific hypotheses for each decision-making level. An example is given in (Figure 1) Interview guides based on these hypotheses were developed and field tested.

Interviews were conducted by anthropologists and were recorded, transcribed, and analyzed using N-Vivo.

\begin{tabular}{|c|c|c|c|c|}
\hline $\begin{array}{l}\text { Vaccine schedule and } \\
\text { delivery that facilitates } \\
\text { maximum coverage } \\
\text { without adversely } \\
\text { affecting other health } \\
\text { service activities. }\end{array}$ & $\begin{array}{l}\text { Health personnel } \\
\text { well informed about } \\
\text { HPV vaccine, } \\
\text { convinced of } \\
\text { benefit, and } \\
\text { motivated }\end{array}$ & $\begin{array}{l}\text { Collaboration } \\
\text { and planning } \\
\text { between Schools } \\
\text { and Health } \\
\text { facility }\end{array}$ & $\begin{array}{l}\text { Quality, source and } \\
\text { consistency of } \\
\text { information about } \\
\text { HPV received by } \\
\text { families, teachers } \\
\text { and health } \\
\text { personnel }\end{array}$ & $\begin{array}{l}\text { Motivational educational } \\
\text { activities aimed at the } \\
\text { population in general, } \\
\text { including health service } \\
\text { personnel, community and } \\
\text { families that provide } \\
\text { explanation of timing of } \\
\text { vaccination in childhood, } \\
\text { natural history of cancer of } \\
\text { the cervix and transmission } \\
\text { of the virus. }\end{array}$ \\
\hline
\end{tabular}

Figure 1: Hypothesis: Health facilities will have higher coverage of the recently reintroduced HPV vaccine in Peru if the following locally determined aspects have been successfully addressed:

\section{Household survey}

We conducted a household survey in the health service catchment areas to provide access to parents' opinions and reasons for acceptance, refusal or discontinuation.

Based on local maps we identified urban areas expected to include 100-150 households, or rural villages, and then randomly selected the survey sites. In addition we added some rural and difficult access areas that had been challenging for the health personnel. In each site starting at a randomly selected point, households were visited in order, to identify school aged girls. As it became obvious that there was a wider than expected range of ages of girls who had been offered the vaccine, and as our purpose was to understand the reactions and motivations of parents with the respect to the vaccination, we expanded the criteria to include all girls who were currently, or the previous year, in 5th or 6th grade of primary school, or were aged 10 or 11 years. We asked their parents about their vaccination status and the reasons.

Ethics: The protocol and instruments were approved by the Institute de Investigation Nutritional ethics committee. Consents were verbal following an appropriate explanation. Individual names were only available to the project team.

\section{Analysis of the data}

\section{Interviews}

Transcriptions were reviewed and the contents summarized into thematic tables and matrices following the principal themes of the interview guide plus other themes identified during the interview or discussion of the results. Within each case, all thematic matrices were analyzed together to triangulate perspectives of all actors (girls, parents, teachers, health workers, and facilities) regarding the factors that facilitate or hinder vaccination feasibility and success. We used $\mathrm{N}$-vivo®for the analysis. We identified direct quotations to illustrate a point and reinforce the findings of the analysis.

\section{Survey}

Survey questions were designed based on previous experience and piloted. We categorized the replies according to original predetermined statements amplified following the results of interviews.

Descriptive statistics of the prevalence of different reasons for vaccination, incomplete vaccination because doses were pending, discontinued vaccination and non-vaccination according to age and school grade were calculated.

\section{Results}

\section{Household survey}

The survey was undertaken between September and December 2015 and included 844households, with girls potentially candidates for the vaccine in the catchment areas of the selected health services. The girls' ages ranged from 9 to 15 years, the majority (65.8\%)aged 10 or 11. In all regions and in both urban and rural areas there was a wide range of ages in 
each grade, for instance in grade 5 girls aged ranged from 9 to 15 . Ten year old girls were in grades ranging from 2 to secondary school, only $59.2 \%(154 / 260)$ were in grade 5. A total of 442 , $52.5 \%$ (95\% CI 48.9-55.6)girls surveyed had received at least 1 HPV vaccine and 199, 23.5\%(95\% CI 20.8-26.5) had received the three doses by the time of the interview. The prevalence of fully vaccinated girls was similar in Ucayali 19.2\% (95\% CI15.4-23.7) and Lima/Callao 21.3\% (95\% CI16.5-27.0) and higher in Cusco $31.1 \%$ (95\% CI 25.8-36.9) where there had been an information campaign supported by an NGO in one district.

The intention was to vaccinate all girls in 5 th grade and those in 6th grade who had not received or completed the vaccine doses the previous year. Of the 335 girls in 5th grade 222, 66.3\% (95\% CI 61.1-71.1) received the 1st dose, 212 in school, 9 in a health facility and 1 at home; $177,52.8 \%(95 \%$ CI 47.5-58.1) received the second dose; and 68, 20.3\%(95\% CI 16.3-24.9) the third dose. Of the 45 girls who had not received the second dose, 28 cases had discontinued and 17 were pending the vaccine. For the third dose 109 girls had not received it, of which 24 had discontinued and 85 were pending. In total $15.5 \%$ of girls had discontinued.

Of the 253 girls in 6th grade, 170, 67.2\% (95\% CI 61.2-72.7) had received the first dose of the vaccine, $145,57.3 \%(95 \% \mathrm{CI}$ 51.2-63.3) the second, and 101, 40.0\%(95\% CI 34.1-46.1)the third. Of these girls $25(14.7 \%)$ had discontinued after the first dose, $29(17.1 \%)$ after the second dose, 4 were pending their second dose and 14 their third dose.

The reasons for vaccination, refusal and discontinuation are discussed in the context of the interview results.

\section{Interviews}

Knowledge and attitudes to the HPV vaccination and the reasons for the failure to be vaccinated, or to complete the doses, were explored in the interviews and focus groups. The findings are arranged according to the hierarchy and timing of the processes involved in the vaccination campaign.

\section{Processes involved in the vaccination strategy}

Vaccine requirements, timing of doses and consequences for logistics and coverage

Although at national level there was a Ministerial document with instructions about eligibility there were difficulties in calculating the requirement for vaccines. Unlike infant vaccines, health services do not have data on the number of eligible girls in their jurisdiction. Estimates were based on information from the National Institute of Information and Statistics but at local level the health regions calculated their vaccine estimates on the number of girls enrolled in 5 th grade in schools. However, children do not necessarily attend school in the district where they live and others may not be attending school. In addition there were also problems calculating the vaccines required using school enrollment data as this information was often not available until up to 2 months after the start of the school year. This information was necessary to order the vaccines from the Ministry in Lima, and so delayed the vaccine supply to health services.
Difficulty in establishing denominators also affected coverage reporting. Health facilities of ten estimated their coverage based on the number of returned consent forms resulting which left no available vaccine if additional girls were identified later.

\section{Coordination with schools}

Although health and education authorities had informed the schools, and in most cases schools were cooperative, in some cases they did not consider the vaccine a priority making coordination more difficult. The vaccine was to be offered in both State and Private schools but in the survey $20 \%$ of eligible girls had not been vaccinated because the vaccine was not offered in their school, usually a private school.

\section{Ensuring opportunity: Reaching and completing the vaccination schedule in all eligible girls}

Lack of opportunity was the most common reason mentioned by $46 \%$ of all parents in the survey and $53 \%$ of parents of fifth graders. Although eligible, $6.2 \%$ of girls in 5 th grade were not vaccinated because they were considered outside the age range. The Directive stated that girls in 5th grade were eligible without specifying age. Girls, not in school, were considered eligible if aged $10 \mathrm{y}$ as this age should coincide with 5 th grade. However, this is often not the case, especially in rural areas. The survey found a wide range of ages in different grades, for instance, of 260 10 year olds $59 \%$ were in 5 th grade, $30 \% 4$ th grade and $4 \% 6$ th grade. Girls in 5th grade ranged from 9 to $15 \mathrm{y}$.

These circumstances created confusion and differences in interpretation of the directive among health personnel, so that in some cases 10 year old girls were vaccinated whether in 5th or 6th grade and in other cases some nurses had concluded that girls aged 9 or 11 were not eligible.

A late start to vaccination was a problem. In one region the first vaccine was sometimes not given until November making it impossible to complete the three doses within the school year. Other reasons for failure to complete doses were because girls did not return to the school the following year, particularly in rural areas and areas with high migration.

Girls aged 10-13 years who missed the school vaccination or had not completed doses, could be vaccinated in their local health center but in some cases, girls who were older than $10 \mathrm{y}$ or girls who had a boyfriends were denied the vaccine based on a misconception that there was a risk of side effects if the girl had started sexual relations.

There were discrepancies between health facilities and regions over vaccination in private schools, and in one region these were excluded, while in another the social security system assumed vaccination in these schools. The survey found $18 \%$ of 113 girls in 5th grade received no vaccine because they were attending excluded schools, mainly private schools.

The situation of children who do not attend schools in the district of residence was not clear, sometimes they were advised to request the vaccine from the health facility nearest to their 
home but others were vaccinated at school. Some girls had been refused the third dose in their health facility because the first two doses had been given in a school that was in a different health jurisdiction. Health personnel expressed their concern that they would run out of vaccines as they had calculated needs based on their local school requirement, others were concerned about lack of control over the number of doses the girl received.

In addition, the vaccine could only be administered once a completed consent was presented although in a few cases nurses, feeling pressurized, vaccinated in the absence of consent.

Nurses made return visits to the school to complete these vaccinations once they had consent but this added additional work and delays as well as increasing the number of times that the class was interrupted.

In the survey, $7 \%$ of parents of fifth graders reported that the girl had not been vaccinated because of lack of signed consent.

When girls were not present for the second or third doses, the health personnel were instructed to do domiciliary visits but in some cases difficulty finding the house meant that vaccination could not be completed.

Domiciliary visits were especially difficult in rural and sparsely populated areas because of the cost and time involved and because agricultural activities meant that children were often not at home. We found some evidence that health personnel prioritized urban schools over rural and in some cases decided not to visit a distant rural area which might take a day's journey with no guarantee that the girl would be present.

The survey also suggested that many girls did not complete the three doses during the school year and this resulted in incomplete vaccination, $18 \%$ of girls who had received their first dose had not received the second dose and parents of 52 fifth graders reported that their daughter had not completed the 3 doses. The most commonly mentioned reason was that the nurse did not return to the school (23\%) followed by the girl not attending school on the day of vaccination (11.5\%). In addition $34.6 \%$ mentioned that they did not know that 3 doses were necessary. Only $15.4 \%$ missed vaccines were for refusal, mostly for fear of side effects although only 1 girl reported an adverse event at the first dose.

\section{Training, coordination and information received by the different actors in the vaccination strategy.}

\section{Health Personnel}

In all three regions an information cascade had been planned starting from the Ministry of Health with presentations to the regional heads of immunization, with the intention that these would then train the vaccination staff in their regions, who would then inform teachers, parents and girls. This system had not been effective in reaching nurses and ancillaries who administered the vaccine who often had little, and sometime mistaken, understanding, of HPV infection, its role in cervical cancer and the importance of the vaccine.
There was variability in the training in the different regions. In one region a local NGO that specializes in cancer prevention had given additional help and provided a variety of attractive materials that were considered very helpful, however, this was unique, and mostly training was varied in content and with no, or very limited, audiovisual aids.

In all regions we interviewed staff who had not received any training, and in general there was a plea for more information and training, especially to ensure homogeneity in the information managed by health personnel.

\section{Education Sector}

HPV vaccination in schools required coordination with the education authorities and schools to provide information to teachers to motivate them as allies in the distribution of the consent forms and the organization of the vaccination.

There was no official planning committee between the Ministries of Education and Health at national level. At regional level coordination depended on the local institutions and was variable. In one region a memo was sent to the education authority informing and requesting their cooperation with facilitating the process in the schools, in another the nurses took advantage of a school health program to give a presentation about the vaccine.

There were mixed reactions from school heads and teachers. Some were unhappy about the interruption of classes and caused delays in the programming. Rapid turnover of staff was another complication.

There was also considerable variation in the information that class teachers received, sometimes the local nurse had visited and informed all involved, but in other cases teachers had received little or no information.

On the other hand, teachers' knowledge and positive attitude proved important for the vaccination and teachers who had been motivated by understanding the importance of the vaccine were influential in parents' decisions to allow their daughter to be vaccinated. However, others mentioned that they had felt they had insufficient knowledge to deal with parents' anxieties about the vaccine.

\section{Parents and Girls}

Girls could only be vaccinated with signed parental consent. Consent forms were distributed to girls, usually by the teachers at school meetings before the vaccination, and girls were required to produce the signed form before they could be vaccinated. This requirement was respected by the nurses and vaccination of girls without consent was deferred to a later date. This meant that nurses had to make a second or third visit to the school and occasionally were unable to vaccinate.

Ideally all parents of eligible girls would have received information about the vaccine and its role in preventing cancer of the cervix preferably from informed sources, but this was not always the case. Limitations included the difficulty of arranging meetings with parents especially if schools were reluctant. Also 
often few parents attended these meetings.

Nurses also found it difficult to make time because of other responsibilities, particularly when the health facilities had introduced an appointment system and the time available for leaving the health service was limited.

There was also a lack of materials to help with training. Sometimes the consent form was the main, and for parents, the only source of information. In general teachers had not received any materials.

In rural areas, particularly in jungle areas, additional difficulties included the logistics and cultural barriers of reaching and undertaking informational activities with schools and parents. In a few cases remote small communities with one or two girls were accorded low priority, or were just not included in the vaccination.

Girls usually received information from the nurses or teachers who felt that the information they could give, particularly in the case of teachers, was important in vaccine acceptance. Some interviewed girls also mentioned that it was the teachers who had explained about the vaccine but many felt that they had not been given sufficient information about the virus and the vaccine.

\section{Communication about the vaccine}

Overwhelmingly the motivation to have accepted the vaccination was to protect against cancer of the cervix, once parents had understood this they were in favor of the vaccination. Between $94 \%$ of parents of fifth graders mentioned this as the reason for accepting the vaccine. In most cases this information came from the nurses. There was a lack of media coverage about the HPV vaccination both at national and local levels with hardly any radio spots or local posters, and only some isolated examples where radio spots, developed by the Ministry of Health were transmitted. There was also a lack of communication materials such as fliers. Lack of funds at all levels, for example to pay for media space, was usually blamed for this.

The household surveys reflect the lack of information reaching the parents about the vaccine, $50 \%$ of parents of unvaccinated girls mentioned that were not vaccinated because of lack of information. Sometimes this was because the girls were not in the appropriate grade, but even when in grade 5, 39\% of parents reported either received no information or not knowing that the vaccine existed. Together with lack of opportunity this was the main reason for not having received the vaccine.

On the other hand, there was also evidence of faith in the schools and health services that worked in favor of the vaccine, a tendency to accept that if the school or the health services offered a vaccine, then it was a good thing.

There was little large scale promotion, in one region there was an official inauguration with local authorities but publicity was not sustained. Some parents in one region mentioned hearing about the vaccination campaign on television, and as a result had taken their daughters to their local health facility but they had not received the vaccine as they were not in the correct age range.
The was also a NGO campaign, very localized, but it provides an example of what can be done as this educational campaign was mentioned by a number of interviewees.

We interviewed some local media who commented that they had not transmitted radio interviews or spots about the HPV vaccine except to comment on the number of people who had died of cervical cancer. National media coverage was limited to a few interviews with experts. Social media was a source of information mentioned by some of those interviewed.

Most health personnel interviewed felt that a national mass media campaign coinciding with each vaccine dose should be implemented and that this should be adapted to the sociolinguistic reality of their areas.

\section{Vaccination process \\ Completion of the three doses}

Each girl who was vaccinated had a vaccine record card to be completed at each vaccination. However it was common for girls to lose or forget these cards for the next dose, so nurses tended to retain the cards only giving them to the girls after the final dose. This presented problems when the girls missed the vaccination at school and presented at health services or were contacted in their homes.

Aware of the challenge of keeping track of the vaccination in school children, the Ministry of Health designed a web based system to track individual's vaccination status. Children in Peru should all have a unique document with an identification number. At the time of vaccination the information was recorded by the nurse on worksheets which were then sent to the immunization service of the regional health service and data entered. The girl's name should match with the DNI but sometimes there was misspelling, incorrect DNIs or incomplete information resulting in missing data. Nurses could access the system but not make changes and correct errors, and there were complaints that this. The delays in data entry meant that the system was of limited help with the follow-up of partially vaccinated girls. Nevertheless there was recognition that this sort of tracking system was necessary to counteract the problems of migration.

\section{Failure to complete the three doses}

The main reasons for incomplete doses was lack of understanding that 3 doses were necessary, and lost opportunities, for instance the nurse did not return to the school ( $23 \%$ of 525 th graders) or the girl did not attend school on the day of the vaccination (11.5\%). In addition $34.6 \%$ mentioned that they did not know that 3 doses were necessary, only $15.4 \%$ were for refusal, mostly for fear of side effects although only 1 girl was reported to have had an adverse event at the first dose.

\section{Refusals}

A search of internet revealed a large number of sites that contain alarming information warning of side effects of vaccines in general and the HPV vaccine in particular. We asked a general question in the household survey about the reason for not 
receiving the vaccine and this was also discussed in interviews, and although some individuals mentioned the adverse mass media coverage, we found little evidence that parents, teachers or health personnel were influenced to refuse or oppose the vaccine because of this information. Only 16 of 47 parents who had refused vaccination mentioned adverse information as a reason.

On the other hand, the main concern expressed by parents and also some teachers was fear of side effects. Some parents mentioned that they preferred to wait until their child was older and their reproductive system more developed. Sterilization was one of the most commonly expressed fears in interviews, but rarely in response to the open question in the survey ( 2 parents). Interviewees commented that refusals were often related to previous adverse experiences or negative perceptions about vaccines, specifically health personnel mentioned fear that the vaccine would cause sterilization; that it would induce the girls to be precociously sexually active; that it would produce fever and convulsions and that the vaccine was "experimental" because it was new, and occasionally there were doubts about the quality of the vaccine because it was given by the government. However, although fear of sterilization was mentioned by health personnel and teachers, it was rarely specifically mentioned by parents when asked why their daughter had not been vaccinated, and only 7 parents expressed a lack of confidence in the health services.

Health personnel commented that when given more information, especially when it was understood that the vaccine was to prevent cancer and that an otherwise expensive vaccine was being offered free, acceptance was high.

For girls the principal fear of vaccination was the injection. If the girls were not convinced by the health advantages and were still fearful nurses had developed strategies to overcome this, for instance leaving the fearful girls until last when, having seen their classmates vaccinated without problems, they usually agreed to be vaccinated. Some nurses also taught the girls how to relax their arms prior to injection, a feature of the demonstration project in Piura.

Overall, we found relatively little evidence of vaccine refusal both in interviews and in the survey. There were some cases where parents mentioned fear of vaccines in general but in these cases nurses had opted to talk directly to parents and had succeeded in convincing them.

\section{Discussion}

Clinical trials have unequivocally shown the efficacy and safety [11] of HPV vaccines in reducing HPV infections, and encouraging results are emerging on the prevention of the cervical lesions that are precursors to cancer of the cervix [2].The acceptability and uptake of the vaccine have been extensively studied in many countries [7] and show that it is possible to reach very high vaccine coverage rates. Fear about the vaccine, especially that it might cause sterilization, and the possibility that it will encourage promiscuity are often reported as being a potential problem but, when parents are adequately informed, especially by personnel in whom they trust, acceptance and uptake of the vaccine is very high $[12,13]$.
The challenge now is to reach and vaccinate girls before sexual debut and exposure to the virus particularly in those countries with the highest rates of cervical cancer, which includes Peru where 1715 women die annually of cervical cancer [1].

School based vaccination has been the preferred choice where most girls attend school and studies in North America, Australia and the UK have shown high uptake and better coverage than health system based strategies $[7,12,14]$. LMIC countries have been slower to introduce the vaccine but school-based vaccination demonstration studies in Peru, Brazil, Uganda, India, Vietnam, and a clinical trial in Tanzania have been promising $[10,13,15$ and 16] although most of these studies have been mainly or exclusively or in urban areas. These demonstration studies have shown high uptakes but have had the advantage of additional supervision and support. A review [17] of the Gardasil access program that supported pilot vaccination in 21 national programs of which 12 included school-based programs also reported high vaccine uptake rates.

These studies have also highlighted the potential challenges facing school based programs; for instance not all adolescents are in school [18], absenteeism and parental refusal are reported albeit in a minority girls [16], migration and scattered sparsely populated areas are particularly challenging in rural areas [10]. School based vaccination may also increase the work load of health personnel [12].

The studies have also identified additional factors important in achieving high coverage. In Brazil this included the "mopping up" arrangements and $20-26 \%$ of doses were "rescue vaccination" [15]. In some countries vaccination through health services has also been successful, for instance in Vietnam and Mexico [14] so that a combined approach may offer the best alternative.

Only one other country, Rwanda, outside the Gardasil access program has published their experience of national introduction of HPV vaccine. They achieved a very high coverage (93\%) with a school based strategy aided by strong political advocacy and coordination with community workers $[19,20]$.

This observational study is one of the first to describe the challenges of implementing a national school-based vaccine program in a LMIC country without outside support and with a diverse geography but high rates of school attendance.

We identified a number of challenges relevant to other countries establishing HPV vaccination though schools. It is important that all schools including private and religious schools participate or alternative strategies are in place for these girls. Some Catholic schools refused to allow HPV vaccination in Ontario when school based vaccination was first introduced [21] and in Tanzania 3 private schools refused to participate [16]. In our study a significant number of girls were not vaccinated because the vaccine was not offered at their school. This was a result of lack of clarity over the policy with regard to private schools in this particular campaign, and has since been corrected, but it is clear that there needs to be a specific policy decision regarding schools outside the public sector. 
We showed that lack of information was a major reason for failure to be vaccinated. Studies have emphasized the importance of information $[22,23]$ and this was a weakness of the campaign in 2015 in Peru when mass media strategies were absent for most of the time. Previous studies [8,24,25 and 26] have emphasized the importance of information and how when information leads to knowledge, coverage rates increase. In a school based study the information received by teachers as well as parents is important. We found that this was variable and there was a general absence of promotional material which would have helped the task of the vaccine nurses and teachers. Anecdotal results of the interviews in Cusco suggest that the materials provided by an NGO were very helpful in promoting the vaccine and this is in keeping with the results of demonstration projects.

We found little evidence, that girls, families had received any information from the media even though there were radio spots, and some materials designed for the purpose. Community involvement has been described to have facilitated vaccine uptake rates but not vaccine acceptance in the GAP countries [17] and other studies also report the benefits of mobilizing community workers and civil society to motivate vaccine uptake [12]. Two community based programs in Peru [27,28] that linked cervical cancer screening with reinforcement of HPV vaccine also reported that community engagement facilitated the vaccination program.

In other countries [29] negative media reports was reported to have created confusion and concern but in Peru we found only sporadic examples where information on internet had adversely influenced parents and/or girls decisions. Nevertheless the threat of adverse reporting is always present especially if unexpected adverse events occur. On the first day of the HPV vaccine demonstration in Piura [10] an unexpected unrelated adverse event occurred that was potentially damaging to the campaign but thanks to an innovative motivational training that had convinced journalists of the benefits there was no adverse media coverage (personal communication).

We encountered many cases of incomplete vaccinations often because the third dose was not completed within the school year, a problem also mentioned by others [12] and it is clearly advantageous if the full vaccine course is completed during the school year. Although current vaccine schedules only require 2 doses [30] the time interval between the first and last dose remains 6 months, and when the school year is 9 months, as in Peru, this is challenging. In this study the inability to complete the vaccination was mostly attributable to late start of vaccination due to delays in completing the register of girls matriculated which then delayed the ordering and arrival of the vaccine. It is desirable to avoid wastage but any strategies that increase availability of the vaccine in sufficient amounts to cover the estimated needs while not risking vaccine expiry dates would be helpful. In Peru there are reliable cold storage facilities with temperature monitoring so that allowing a degree of overestimation of demand would not necessarily risk wastage.

We identified a wide range of ages in school grades and this compounded confusion as to the eligibility by age or grade. A similar situation was reported in Uganda [31]. A study in Tanzania [16] and a review [7] concluded that grade based vaccination was associated with higher coverage and completion. In general grade rather than age also involves fewer teachers and easier follow-up [32] but makes it even more important to complete vaccination within the school year as girls may not necessarily pass to the next grade or may change school and be missed [7].

The consent process also caused delays, as girls could not be vaccinated without signed consent. This problem is discussed in other reviews [33] that emphasize that the content of the form and timing of distribution are important but considerable effort by teachers and vaccinators may be needed to obtain a high rate of returned forms. There is a need to test alternative ways of informing and obtaining the consent of parents, for instance consenting at the time of matriculation or other opportunities when parents have to go to the schools. In 2017 the Peruvian Ministry of Health published the consent form together with information about the vaccine in national newspapers as an additional strategy to overcome these difficulties. Some countries have introduced opt-out consent procedures that have increased coverage for vaccination accessed 25 Feb 2018).

Migration and attendance at schools outside the girl's residential district add logistic complications and additional work burden to the follow-up of girls who missed doses. In these context web-based national information systems, as was developed in Peru, have potential but are not necessarily straight forward. A system for correcting errors needs to be in place, and the information needs to be available to all potential users. Some of the problems with the system in Peru could be attributed to "teething problems" and will be improved but it is clear that a form of tracking system is necessary particularly if there is a mixed offer of school and health service vaccination. Giving vaccine certificates or cards was not efficient as they got lost. In some places community involvement has helped with follow-up but these programs were effective in relatively localized settings.

\section{Strengths of the study}

The study was informed by mixed methods: interviews of a variety of actors in three very different contexts and a household survey to reach parents and get firsthand information on reasons for non-vaccination. We suggest that this provides helpful information for other countries/health services organizing vaccination of school aged children

\section{Weaknesses of the study}

We only included 3 areas of the country and within the regions, districts that were selected by the health services as contrasting relatively high and low coverage. Our survey was not designed as a coverage survey but as a complement to the qualitative methods designed to capture the range and extent of barriers to vaccination and the perceptions of parents.

\section{Conclusion}

We describe some of the factors that facilitated vaccination 
and the barriers that contributed to non-vaccination and incomplete doses in nationally implemented school based vaccination strategy.

The main barriers were lack of information and lack of opportunity. Publicity stressing the health benefits, safety, and importance of three doses given by an informed and motivated media that will reach parents and children is needed at the beginning of the campaign [30]. In the few cases of refusal by the adolescent girls fearful of injection pain relaxation exercises can help reduce this and are simple to teach (personal communication).

We have identified that there were many logistic challenges and difficulties in organizing and completing the vaccination in a school based system, In particular the problems of accurately determining, at the beginning of the school year, the number of vaccines needed and organizing parental consent result in delays in the start and makes it difficult to complete the doses when the gap between doses is 6 months within a 9 month school year.

Coordinating with the schools so that opportunities such as school matriculation or other occasions when parents necessarily have to visit the school might have helped ensure that consent did not delay the vaccination.

Even if there can be a timely start to vaccination it likely that there will be a need for back up or "mopping up strategies" as was described in Mexico [15]. It will be necessary to maintain the possibility of health service vaccination. In a mixed system the ability to identify which children have been vaccinated and with how many doses is challenging. Community engagement, where possible, can be harnessed to increase vaccine coverage and completion but may not always be available. Web based systems such as that developed for the HPV vaccination in Peru may be possible but only in countries where children have unique identification documents.

We have described some of the successes and difficulties of implementing a school-based strategy for HPV vaccine in a middle income Latin American country. Some of the difficulties are already being addressed in Peru but we believe that the experience of this campaign is relevant to other governments facing the challenge of implementing this life saving vaccine in the countries where it is most needed.

\section{References}

1. Bruni L, Barrionuevo-Rosas L, Albero G, Serrano B, Mena M and Gómez D, et al. Human Papillomavirus and Related Diseases in Peru. Summary Report 15 December 2016. ICO Information Centre on HPV and Cancer (HPV Information Centre).

2. Garland SM, Kjaer SK, Muñoz N, Block SL, Brown DR and DiNubile MJ, et al. Impact and Effectiveness of the Quadrivalent Human Papillomavirus Vaccine: A Systematic Review of 10 Years of Realworld Experience. Clin Infect Dis. 2016;63(4):519-527. doi: 10.1093/ cid/ciw354

3. Baldur-Felskov B, Dehlendorff C, Munk C, Kjaer SK. Early impact of human papillomavirus vaccination on cervical neoplasia-nationwide follow-up of young Danish women. J Natl Cancer Inst. 2014;106(3):460. doi:10.1093/jnci/djt460

4. Gertig DM, Brotherton JM, Budd AC, Drennan K, Chappell G and Saville AM. Impact of a population-based HPV vaccination program on cervical abnormalities: a data linkage study. BMC Med. 2013;11:227. doi: 10.1186/1741-7015-11-22

5. Hariri S, Bennett NM, Niccolai LM, Schafer S, Park IU and Bloch KC, et al. Reduction in HPV 16/18-associated high grade cervical lesions following HPV vaccine introduction in the United States - 2008-2012. Vaccine. 2015;33(13):1608-1611. doi: 10.1016/j. vaccine.2015.01.084

6. Das JK, Salam RA, Arshad A, Lassi ZS, Bhutta ZA. Systematic review and meta-analysis of interventions to improve access and coverage of adolescent immunizations. J Adolesc Health. 2016;59(4):40-48. doi: 10.1016/j.jadohealth.2016.07.005

7. Paul P, Fabio A. Literature review of HPV vaccine delivery strategies: considerations for school- and non-school based immunization program. Vaccine. 2014;32(3):320-326. doi:10.1016/j. vaccine.2013.11.070

8. Hanson CM, Eckert L, Bloem P, Cernuschi T. Gavi HPV Programs: Application to Implementation. Vaccines. 2015;3(2):408-419. doi:10.3390/vaccines3020408

9. Farias CC, Jesus DV, Moraes HS, Buttenbender IF, Martins IS, et al. Factors related to non-compliance to HPV vaccination in RoraimaBrazil: a region with a high incidence of cervical cancer. BMC Health Serv Res. 2016;16:417. doi: 10.1186/s12913-016-1677-y.

10. Penny M, Bartolini R, Mosqueira NR, LaMontagne DS, Mendoza MA and Ramos I, et al. Strategies to vaccinate against cancer of the cervix: feasibility of a school-based HPV vaccination program in Peru. Vaccine. 2011;29(31):5022-5030. doi: 10.1016/j. vaccine.2011.04.078

11. Castle PE Maza M. Prophylactic HPV vaccination: past, present, and future. Epidemiol Infect. 2016;144(3):449-468. doi:10.1017/ S0950268815002198

12.WigleJ,CoastE,Watson-JonesD.Humanpapillomavirus(HPV)vaccine implementation in low and middle-income countries(LMICs): Health system experiences and prospects. Vaccine.2013;31(37):3811-3817. doi.org/10.1016/j.vaccine.2013.06.016

13.LaMontagne DS, Barge S, Le NT, Mugisha E, Penny ME, Gandhi S, et al. Human papillomavirus vaccine delivery strategies that achieved high coverage in low- and middle-income countries. Bull World Health Organ. 2011;89:821-830. doi:10.2471/BLT.11.089862

14. Markowitz LE, Tsu V, Deeks SL, Cubie H, Wang SA and Vicari AS, et al. Human Papillomavirus Vaccine Introduction - The First Five Years. Vaccine. 2012;30(5):139-148. doi.org/10.1016/j. vaccine.2012.05.039

15.Fregnani JHTG, Carvalho AL, Eluf-Neto J, Ribeiro Kde C, Kuil Lde M and da Silva TA, et al. A School-Based Human Papillomavirus Vaccination Program in Barretos, Brazil: Final Results of a 
Demonstrative Study. PLoS ONE. 2013;8(4):62647. doi:10.1371/ journal.pone.0062647

16. Watson-Jones D, Baisley K, Ponsiano R, Lemme F, Remes P and Ross D, et al. Human papillomavirus vaccination in Tanzanian schoolgirls: cluster-randomized trial comparing 2 vaccine-delivery strategies. ] Infect Dis. 2012;206(5):678-686. doi:10.1093/infdis/jis407

17. Ladner J, Besson M, Rodrigues M, Audureau E, Saba J. Performance of $21 \mathrm{HPV}$ vaccination programs implemented in low and middleincome countries, 2009-2013. BMC Public Health 2014;14:670. doi: $10.1186 / 1471-2458-14-670$

18. Nutunen K, Lehtinen J, Namujju P, John Sellors, and Matti Lehtinen. Aspects of Prophylactic Vaccination against Cervical Cancer and Other Human Papillomavirus-Related Cancers in Developing Countries. Infectious Diseases in Obstetrics and Gynecology. 2011; doi:10.1155/2011/675858

19. Binagwaho A, Wagner CM, GateraM, Karema C, Nutt CT and Ngabo F. Achieving high coverage in Rwanda's national human papillomavirus vaccination programme. Bull World Health Organ 2012;90(8):623-628. doi:10.2471/BLT.11.097253

20. Gatera M, Bhatt S, Ngabo F, Utamuliza M, Sibomana H and Karema C, et al. Successive introduction of four new vaccines in Rwanda: High coverage and rapid scale up of Rwanda's expanded immunization program from 2009 to 2013. Vaccine 2016;34(29):3420-3426. doi org/10.1016/j.vaccine.2015.11.076

21.Wilson SE, Karas E, Crowcroft NS, Bontovics E, Deeks SL. Ontario's School-based HPV Immunization Program: School Board Assent and Parental Consent. Can J Public Health. 2012;103(1):34-39.

22.Vermandere H, Naanyu V, Mabeya H, Vanden Broeck D, Michielsen $\mathrm{K}$ and Degomme 0. Determinants of Acceptance and Subsequent Uptake of the HPV Vaccine in a Cohort in Eldoret, Kenya. PLoS ONE 2014;9(10):109353. doi:10.1371/journal.pone.0109353

23.Bartolini RM, Winkler JL, Penny ME, LaMontagne DS. Parental Acceptance of HPV Vaccine in Peru: A Decision Framework. PLOS ONE. 2012;7(10):48017. doi:10.1371/journal.pone.0048017

24.Wang WCW, Ma Y, Wang X, Zou H, Zhao F and Wang S, et al. Acceptability of human papillomavirus vaccine among parents of junior middle school students in Jinan, China. J vaccine. 2015;33(22):2570-2576. doi.org/10.1016/j.vaccine.2015.04.010

25.Sanchez Anguiano LF, Lechuga Quiñones AM, Milla Villeda RH, Lares Bayona EF. Knowledge and acceptance of vaccine against human papillomavirus among mothers of students from Durango city, Mexico. Ginecol Obstet Mex. 2013;81(2):77-85.
26.Winkler JL, Wittet S, Bartolini RM, Creed-Kanashiro HM, Lazcano-Ponce E and Lewis-Bell K, et al. Determinants of Human Papillomavirus Vaccine Acceptability in Latin America and the Caribbean. Vaccine. 2008;26(11):73-79. doi.org/10.1016/j. vaccine.2008.05.027

27. Abuelo CE, Levinson KL, Salmeron J, Carlos Vallejos Sologuren, Maria Jose Vallejos Fernandez, and Jerome L. Belinson. The Peru Cervical Cancer Screening Study (PERCAPS): The Design and Implementation of a Mother/Daughter Screen, Treat, and Vaccinate Program in the Peruvian Jungle. J Community Health. 2014;39(3):409-415. Doi:10.1007/s10900-013-9786-6

28.Levinson KL, Abuelo C, Chyung E, Salmeron J, Belinson SE and Sologuren CV, et al, The Peru Cervical Cancer Prevention Study (PERCAPS): Community Based participatory Research in Manchay, Peru. Int J Gynecol Cancer. 2013;23(1):141-147. doi:10.1097/ IGC.0b013e318275b007

29. La Vincente SF, Mielnik D, JenkinsK, Bingwor F, Volavola L and Marshall H, et al. Implementation of a national school-based Human Papillomavirus (HPV) vaccine campaign in Fiji: knowledge, vaccine acceptability and information needs of parents. BMC Public Health. 2015;15:1257. doi 10.1186/s12889-015-2579-3

30. Meites E, Kempe A, Markowitz LE. Use of a 2-Dose Schedule for Human Papillomavirus Vaccination - Updated Recommendations of the Advisory Committee on Immunization Practices. MMWR Morb Mortal Wkly Rep. 2016;65(49):1405-1408. doi:10.15585/mmwr. mm6549a5

31. Mugisha E, LaMontagne DS, Katahoire AR, Murokora D, Kumakech E and Seruyange $\mathrm{R}$ et al. Feasibility of delivering HPV vaccine to girls aged 10 to 15 years in Uganda. African Health Sciences. 2015;15(1):33-41. doi: 10.4314/ahs.v15i1.5

32.Perman S, Turner S, Ramsay AIG, Baim-Lance A, Utley M, Fulop NJ et al. School-based vaccination programmes: a systematic review of the evidence on organization and delivery in high income countries. BMC Public Health. 2017;17:252. doi 10.1186/s12889-017-4168-0

33.Fisher WA. Understanding Human Papillomavirus Vaccine Uptake. Vaccine. 2012;30(5):149-156. doi.org/10.1016/j. vaccine.2012.04.107

34. Resolución Ministerial. 255-2015.

35.WHO. Considerations regarding consent in vaccinating children and adolescents between 6 and 17 years old. WHO reference number: $2014 ; 8$. 